\title{
Experimental investigation of impinging jet erosion on model cohesive granular materials
}

\author{
Florian Brunier-Coulin ${ }^{1, *}$, Jean-Loup Sarrat ${ }^{1}$, Pablo Cuéllar ${ }^{1}$, and Pierre Philippe ${ }^{1}$ \\ ${ }^{1}$ IRSTEA, 3275 route de Cézanne, CS40061, 13182 Aix-en-Provence Cedex 5, France
}

\begin{abstract}
Erosion of soils affects both natural landscapes and engineering constructions as embankment dams or levees. Improving the safety of such earthen structures requires in particular finding out more about the elementary mechanisms involved in soil erosion. Towards this end, an experimental work was undertaken in three steps. First, several model materials were developed, made of grains (mostly glass beads) with solid bridges at particle contacts whose mechanical yield strength can be continuously varied. Furthermore, for most of them, we succeeded in obtaining a translucent system for the purpose of direct visualization. Second, these materials were tested against surface erosion by an impinging jet to determine a critical shear stress and a kinetic coefficient [2,3]. Note that an adapted device based on optical techniques (combination of Refractive Index Matching and Planar Laser Induced Fluorescence [3]) was used specifically for the transparent media. Third, some specifically developed mechanical tests, and particularly traction tests, were implemented to estimate the mechanical strength of the solid bridges both at micro-scale (single contact) and at macro-scale (sample) and to investigate a supposed relationship with soil resistance to erosion.
\end{abstract}

\section{Introduction}

Hydraulic erosion of soils is a phenomenon due to the action of water on the detachment and transport of elementary soil particles. The physical process by which the fluid flow causes the dislodgment of primary particles is a key problem for many fields. In civil engineering, Foster and co-workers [1] suspected that $90 \%$ of embankment dam failures are due to erosion while, according to the International Commission Of Large Dams, an estimated proportion of $75 \%$ of dams are built in sand, clay or muddy sediments, and are consequently threatened by the risk of erosion. As a consequence, the stability and durability of earthen hydraulic structures depend on the response of soil against hydrodynamic stresses. In France there are on average approximately one failure of dams and one failure of protection dikes per year. In order to enhance the safety of these structures, it is important to comprehend better the elementary mechanisms involved during erosion processes at the surface of a cohesive material.

Different erosion laws can be found in the literature. Most of them involve two erosion parameters accounting for the erosion threshold and the kinetics of erosion. These laws are usually based on a mean hydrodynamic shear stress related to the fluid velocity and pressure gradient, both of them averaged in time and space. The erosion parameters are a priori inherent to the material and can be estimated experimentally by adjusting specifically an erosion law with data obtained from specific erosion tests. However, it is difficult to identify a clear correlation between these parameters and other more common soil properties.

A parametric study based on model materials is proposed here, to seek for a clearer relation between common soil properties and resistance against erosion. We will first present the model materials, based on glass beads with a cohesion made of solid bonds, and then the erosion and mechanical tests used in this study. Finally, the results of this analysis are presented and discussed in the last section.

\section{Model materials}

\subsection{Granular sample}

The model material used in the present study is made of millimetric glass beads with inter-particle solid bonds. The spherical glass beads were provided by SiLiBeads with two distinct types of glass. Some samples are based on silica glass beads with a bulk density $\rho_{s}=2500 \mathrm{~kg} \cdot \mathrm{m}^{-3}$, and with diameters $d$ ranging either between 2.85 and $3.30 \mathrm{~mm}$, or between 0.75 and $1 \mathrm{~mm}$. For purpose of index-matching as discussed later, borosilicate glass beads with a lower refractive index $n=1.472$, a bulk density $\rho_{b}=2230 \mathrm{~kg} \cdot \mathrm{m}^{-3}$ and a diameter $d=3 \pm 0.02 \mathrm{~mm}$ are also used.

\subsection{Solid bridges}

To obtain a cohesive sample, we chose to mix each granular sample with a liquid matrix so as to bond the beads with capillary bridges. With specific liquids like paraffin or resin, it is possible to obtain solid bridges after cooling or drying. In this study, different volumes of transparent liquid resin (provided by Syntilor) are mixed with the beads in a rectangular box of $20 \times 20 \times 10 \mathrm{~cm}^{3}$, before drying at $60^{\circ} \mathrm{c}$ during 7 days to obtain solid bonds. A typical solid bridge between two beads with $d=3 \mathrm{~mm}$ is shown on Figure 1 .

The protocol used to prepare the cohesive samples enables to assume a random loose packing with, typically, a volume fraction $\phi=0,6$ and a number of bonds per particle $z=6[5]$. 


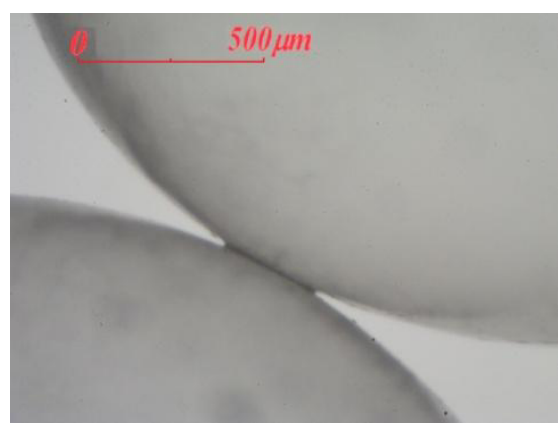

Fig. 1. Typical solid bridge of resin between two glass beads of diameter $3 \mathrm{~mm}$.

\section{Experimental set up and protocol}

\subsection{Index matched round impinging jet}

One of the most popular erosion test is the JET test proposed by [2], used in situ or ex situ in laboratory. As sketched in Figure 2, the set-up developed in this study is adapted from the JET test to fulfil the requirements of the optical techniques used to visualise inside the material. The cohesive sample is implemented under a cell of $40 \mathrm{~cm}$ high and a cross section area $10 \times 20 \mathrm{~cm}^{2}$. The cell is slowly saturated with an oil mixture made of light mineral oil supplied by Sigma-Aldrich $(90 \%$ in mass) and immersion oil supplied by Cargille (10\% in mass). This mixture has the same refractive index than the borosilicate glass beads with a dynamic viscosity $\mu_{f} \approx 0.28 c P$ and a bulk density $\rho_{f}=847 \mathrm{~kg} \cdot \mathrm{m}^{-3}$.

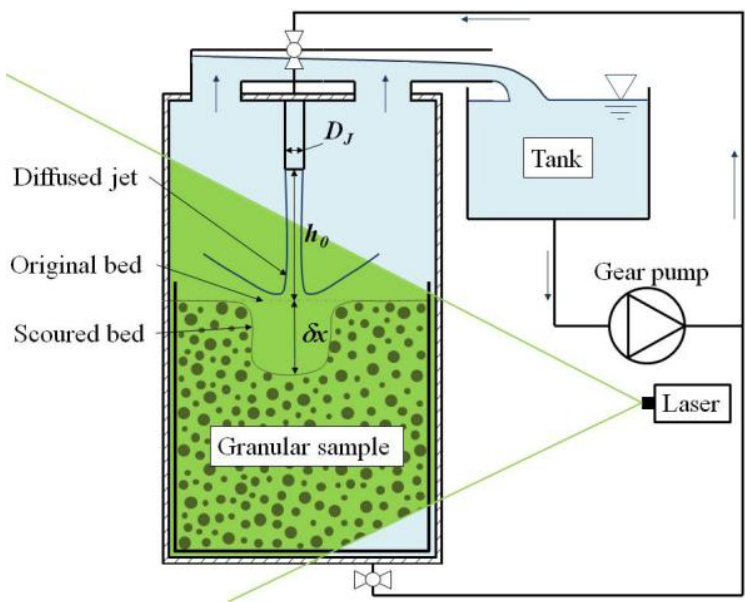

Fig. 2. Index matched erosion tests with a round impinging jet.

The upper side of the cell includes two outlets to create an overflow system, and a central inlet connected to a gear pump. The inlet is composed of a metal tube of $4 \mathrm{~cm}$ length and $5 \mathrm{~mm}$ of inner diameter $D_{J}$ to generate a downward vertical immersed jet at a controlled flow rate supplied by the pump from a liquid reservoir tank.

To visualise the erosion process, a fluorescent dye (Nile Red supplied by Fluka) is added to the immersion fluid, and a planar laser of wavelength $532 \mathrm{~nm}$, inside the dye fluorescence spectrum, is placed in the jet axis.
Images are recorded through a high pass optical filter at $580 \mathrm{~nm}$ by a digital camera with a frame rate up to 20 images per second.

To analyse the erosion threshold and the evolution of the crater depth $(\delta x)$, the jet injection velocity is slowly increased from 0 to $10 \mathrm{~m} / \mathrm{s}$ in almost $1 \mathrm{~min}$. This slow acceleration ramp enables to assume a quasi-steady fully developed jet (i.e. local velocity on the granular surface can be calculated directly from the injection velocity).

\subsection{Mechanical traction test}

To quantify the cohesive forces contributing to the resistance against erosion, two mechanical tests have been developed to measure the mechanical resistance at different scales. For the larger beads $(>1 \mathrm{~mm})$, it is possible to extract from the cohesive sample two beads still bonded together. One bead is glued to a movable deck when the upper bead is held up with a micrometric clamp connected to a scale (see Figure 3 on the left). Then the deck is slowly moving downward and the scale measurement is recording until the failure of the bond. The resulting normal traction force of the solid bridge at failure is noted $F_{t}{ }^{*}$.
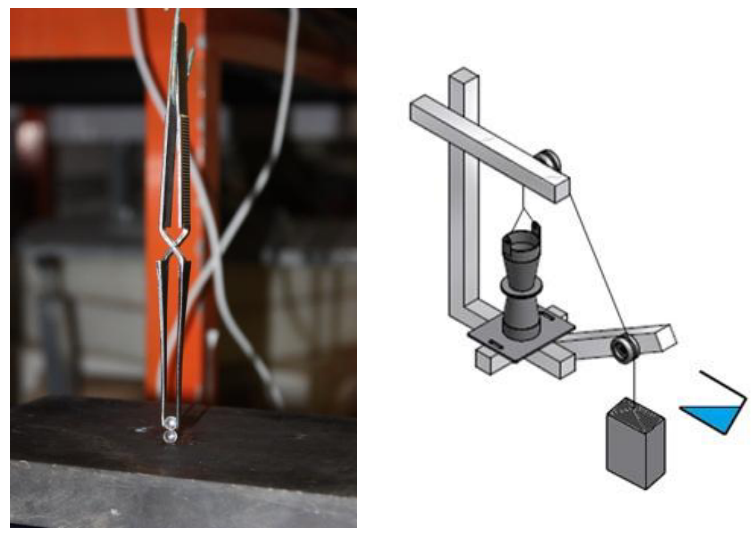

Fig. 3. Micro-scale traction test between two beads of $3 \mathrm{~mm}$ (left) and macro-scale traction test on a representative volume of beads of $1 \mathrm{~mm}$ (right).

For the smaller beads $(\leq 1 \mathrm{~mm})$, a second device enables to measure the global de-cohesion stress on a representative volume of cohesive soil. Before drying, the beads mixed with the liquid resin are set in place inside two conical cylinders as showing in Figure 3. After solidification of the capillary bridges (see section 2.2), the load is slowly increased vertically on the upper part until ultimate separation of both parts. The resulting critical force is divided by the circular failure section to calculate a normal stress of de-bonding named $\tau_{t}{ }^{*}$.

\section{Results and analysis}

\subsection{Experimental results}




\subsubsection{Erosion tests}

To compare the impact of cohesion according to the different bead sizes, an adapted variable seems to be the mean thickness of liquid resin (e), calculated as the ratio of the volume of resin to the total bead surface. Figure 4 shows as the critical injection velocity generating first beads removal $\left(U_{J}{ }^{*}\right)$ increases with $e$ for the different bead sizes.

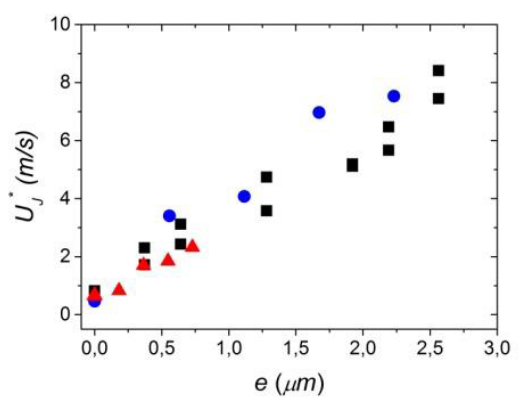

Fig. 4. Critical jet injection velocity $\left(U_{J}^{*}\right)$ versus mean resin thickness (e). Blue circles, black squares, and red triangles stand for borosilicate glass beads of $3 \mathrm{~mm}$, silica glass beads of $3 \mathrm{~mm}$, and silica glass beads of $1 \mathrm{~mm}$ respectively.

From the critical injection velocities, it is possible to calculate the local velocity in the jet centreline, $u_{0}{ }^{*}$, at the distance $h_{0}$ between the nozzle and the granular bed by a theoretical relation given by [6] for a self-similar laminar jet model and validated for the present jet configuration:

$$
u_{0}^{*}\left(h_{0}\right)=U_{J}^{*} \frac{R e_{J}^{*}}{8} \frac{D_{J}}{\left(h_{0}+0.048 R e_{J}^{*}\right)}
$$

where $U_{J}{ }^{*}$ is the injection velocity and $R e_{J}{ }^{*}=\frac{U_{J}{ }^{*} D_{J}}{v_{f}}$ with $v_{f}$ the kinematic viscosity of the fluid.

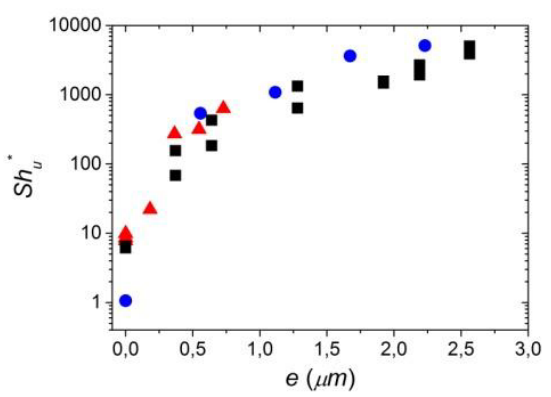

Fig. 5. Critical Shields number $\left(S h_{c l}{ }^{*}\right)$ as a function of the mean thickness of resin (e) with the same symbols as used in Figure 4.

Then, following a previous study by [7], an inertial cohesion-less Shields number $S h_{c l}$ is used to account for the erosion threshold of a grain without cohesion and compares the fluid stress, expressed by $\rho_{f} u_{0}{ }^{2}$, and the buoyant weight of the particle without any shape factor. Consequently, the resulting critical value reads:

$$
S h_{c l}{ }^{*}=\frac{\rho_{f} u_{0}{ }^{2}}{\Delta \rho g d}
$$

with $\Delta \rho$ the difference between the particle density and the fluid density.

Figure 5 shows a strong increase of the Shields number with $e$ over more than three orders of magnitude, highlighting a great influence of adhesive or cohesive forces on the Shields number as expected [8].

\subsubsection{Mechanical resistance of the bonds}

The traction tests realised at different scales enable to quantify the ratio between the buoyant weight of the bead and the critical failure force of the solid bridges by a generalised Bond number. Dimensionally this number can be expressed either from the local traction force or from the global traction stress $\left(\mathrm{Bo}_{m}\right.$ or $\mathrm{Bo}_{M}$ respectively) as:

$$
\begin{aligned}
& B o_{m}=\frac{\Delta \rho g d^{3}}{F_{t}{ }^{*}} \\
& B o_{M}=\frac{\Delta \rho g d}{\tau_{t}{ }^{*}}
\end{aligned}
$$

The Bond numbers calculated from equations 3 and 4 for beads of 1 and $3 \mathrm{~mm}$ almost collapse as shown in Figure 6 when plotting against $e$. The decrease of $B o$ with $e$ (in $\mu \mathrm{m})$ can be fitted by an inverse relation as represented in dashed line on Figure 6. This relation can be used for purpose of Bond number interpolation.

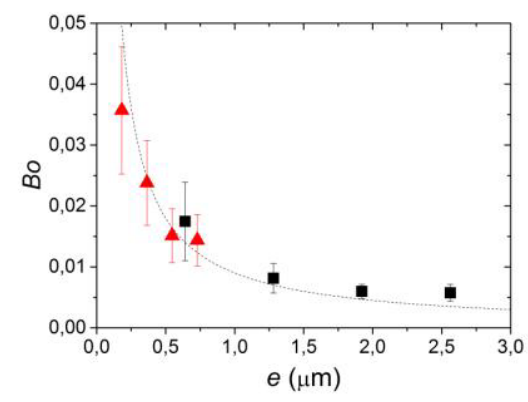

Fig.6. Evolution of the Bond number as a function of the mean thickness of resin with the same symbols as used in Figure 4.

\subsection{Discussion}

The traction stress measured at micro and macroscale can be used to model the impact of the cohesion on the erosion threshold, additionally to weight and friction. A simple assumption is to simply sum the buoyant weight and the cohesive stress in the Shields 
formulation. The resulting generalised Shields number is then expressed via the Bond number as:

$$
S h=\frac{\tau_{f}}{\tau_{m}(1+B o)}
$$

where $\tau_{m}$ is the mechanical traction stress and $\tau_{f}$ is the fluid stress.

The critical cohesion-less Shields number $S h_{c l}{ }^{*}$ and generalised Shields number $S h^{*}$ are represented on Figure 7 as a function of the particle Reynolds number $R e_{p}=\frac{u_{0}{ }^{*} d}{v_{f}}$. This Figure shows that the new formulation of the Shields number enables to rationalize the data, in spite of the dependency between the inertial Shields and the Reynolds number due to the fluid velocity.

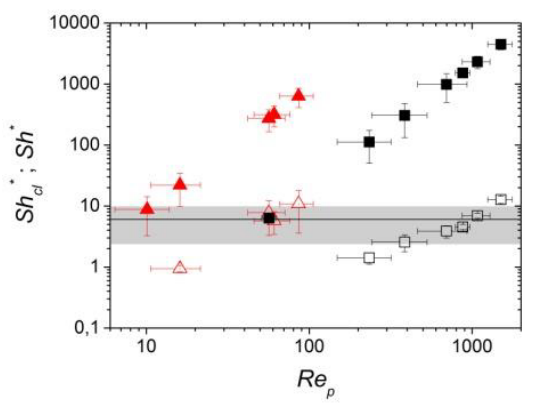

Fig.7. Cohesion-less Shields number (filled symbols) and cohesive Shields number (open symbols) as a function of the Reynolds Particular for both beads size (red triangle $d \approx 1 \mathrm{~mm}$ and black square $d \approx 3 \mathrm{~mm}$ ).

The values of the generalised Shields number can be averaged to obtain a value of $S h^{*}=3.0 \pm 1.9$ (black curve and grey area in Figure 7). This value is in good agreement with those obtained for cohesion-less beads with an inertial Shields number either by us or by [7] with a planar jet.

In the case of Bond numbers far below 1 (i.e. $\left.\tau_{m} \gg \Delta \rho g d\right)$, the relation between $S h_{c l}{ }^{*}$ and $S h^{*}$ can be expressed:

$$
S h_{c l}{ }^{*}=S h^{*}\left(1+\frac{1}{B o}\right) \sim \frac{S h^{*}}{B o}
$$

Accordingly, Figure 8 shows the dependency between $S h_{c l}{ }^{*}$ and 1/Bo. The slope equal to the averaged value of $S h^{*} \sim 3$ is in approximate agreement with most of the data.

\section{Conclusion}

The development of model cohesive granular materials used in parallel with an index matched impinging jet and traction tests at micro and macro-scale allowed to propose a generalised expression of the Shields number for both cohesive and cohesion-less materials in good agreement with the present experimental data.
A first perspective is to better understand the link between micro-scale and macro-scale in realising local and global traction tests with the same material. For that, the size of the set-up has to be adapted to the bead diameters. Finally, this study was realised with solid bounding and a specific size of beads. The perspectives for a future work are to expand this study to a larger range of particle sizes and shapes and to different kinds of bonds like capillary bridges with a viscous fluid or a synthetic clay.

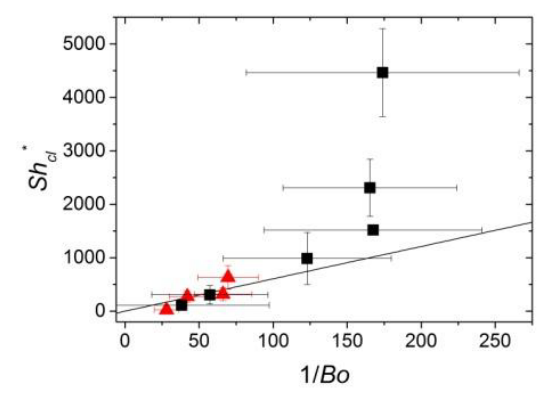

Fig. 8. Cohesion-less Shields number as a function of $1 / B o$ for silica beads of 1 and $3 \mathrm{~mm}$.

\section{Acknowledgment}

We acknowledge financial support from the region PACA (Provence-Alpes-Côte d'Azur).

\section{References}

1. M. Foster, R. Fell, and M. Spannagle, The statistics of embankment dam failures and accidents. Can. Geotech. J. 37:1000-1024 (2000)

2. G. J. Hanson and K. R. Cook, Apparatus, test procedures, and analytical methods to measure soil erodibility in situ. Applied Engineering in Agriculture 20(4):455-462 (2004)

3. E. Partheniades, Erosion and deposition of cohesive soils. ASCE 91(HY1): 105-139 (1965)

4. P. Philippe and M. Badiane, Localized fluidization in a granular medium. Phys. Rev. E 87, 042206 (2013)

5. J.-Y. Delenne, F. Soulié, M. S. El Youssoufi and F. Radjaï, From liquid to solid bounding in cohesive granular media. Mechanics of Materials 43:529-537 (2011)

6. A. Revuelta, A. L. Sánchez and A. Liñán, Confined axisymmetric laminar jets with large expansion ratios. J. Fluid Mech. 456:319-352 (2002)

7. S. Badr, G. Gauthier and P. Gondret, Erosion threshold of a liquid immersed granular bed by an impinging plane liquid jet. Phys. Fluids 26:023302 (2014)

8. S. E. Coleman and V. I. Nikora, A unifying framework for particle entrainment. Water Resources Research 44:W04415 (2008) 